\title{
Influence of Recoil Effect and Surface Excitations on the Inelastic Mean Free Paths of Electrons in Polymers
}

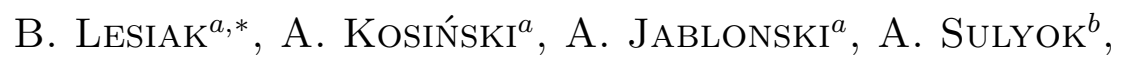 \\ G. Gergely ${ }^{b}$, J. Tóth ${ }^{c}$ And D. VARGA ${ }^{c}$
}

${ }^{a}$ Institute of Physical Chemistry, Polish Academy of Sciences

Kasprzaka 44/52, 01-224 Warszawa, Poland

${ }^{b}$ Institute for Technical Physics and Materials Science

Hungarian Academy of Sciences

P.O. Box 49, 1545 Budapest, Hungary

${ }^{c}$ Research Institute for Nuclear Physics ATOMKI

Hungarian Academy of Sciences

P.O. Box 51, 4001, Debrecen, Hungary

(Received July 11, 2005; revised version January 20, 2006;

in final form March 14, 2006)

In this work, the influence of recoil effect and surface excitations on the inelastic mean free paths for polythiophenes is investigated. The inelastic mean free paths of electrons in polythiophenes are measured with the elastic peak electron spectroscopy method using the Ag standard and the electron elastic scattering cross-sections from the database NIST 3.1 in the electron kinetic energy range 200-5000 eV. The Monte Carlo model is applied for evaluating the electron backscattering intensities from the polymers and the $\mathrm{Ag}$ standard, as well as for evaluating electrons quasi-elastically backscattered from atoms of different atomic numbers (the recoil effect). The surface excitation corrections are accounted for using the formalism of Chen, with the material parameters for polythiophenes evaluated from the elastic peak electron spectroscopy method. Deviations due to recoil effect and surface excitations to the inelastic mean free paths are compared and discussed. Correction to the inelastic mean free paths due to recoil effect is considerable but is smaller, however, than the correction due to surface excitations. Accounting for recoil effect and surface excitations leads to improvement of the inelastic mean free paths, as compared to the inelastic mean free paths resulting from the predictive formulae of Gries.

PACS numbers: 82.35.Cd, 82.80.Pv, 87.16.Ac

*corresponding author; e-mail: blo@ichf.edu.pl 


\section{Introduction}

The inelastic mean free path (IMFP), defined as the average distance that an electron travels between successive inelastic collisions [1], is a crucial parameter for quantitative analysis by electron spectroscopy methods, Auger electron spectroscopy (AES), and X-ray photoelectron spectroscopy (XPS). This parameter can be obtained from different sources. The IMFPs can be obtained from the elastic peak electron spectroscopy (EPES), calculated from the optical data, and from predictive formulae. Extensive comparisons have been made in a review by Powell and Jablonski [2]. For determining the IMFPs in polymers, the predictive formulae such as the TPP-2M formula of Tanuma et al. [3], the G1 formula of Gries [4] and the quantitative structure-property relationship proposed by Cumpson [5] can be used. The IMFPs obtained from the EPES method have been published for selected conductive polymers, such as polyacetylenes, Pd doped polyacetylenes $[6,7]$, polyanilines $[8,9]$, and polythiophenes [10]. Discrepancies between measured [6-10] and predicted IMFPs $[3,4]$ have been observed. Part of the deviations can be ascribed to some problems in determination of IMFPs in polymers by the EPES method. Variation in surface composition and density may occur during measurement due to X-ray or electron beam interactions with the polymers. Atomic concentration of hydrogen cannot be estimated by AES and XPS. Accuracy of IMFPs derived from the EPES method depends on statistical and systematic errors, related to the electron elastic backscattering measurement, the reliability of the models simulating electron transport for the solid, the accuracy of input parameters needed for the electron transport model, such as the atomic composition and density of the sample, the IMFP of electrons in the standard, and the accuracy of the differential cross-sections for elastic scattering of electron in the solid.

The Monte Carlo (MC) algorithm used for simulating electron transport in a solid neglects recoil effect [11-14] and surface excitations [15-23]. The IMFPs for polythiophenes measured by EPES using a high resolution analyser have already been published [10]. Statistical and systematic errors have been estimated for the experimental IMFPs [10]. The aim of the present work is improving the accuracy of IMFPs for polythiophenes derived using the EPES method and Ag standard material by applying new differential cross-sections for electron elastic scattering, and introducing the corrections due to recoil effect, particularly for hydrogen atoms, and corrections due to surface excitations.

\section{Elastic peak electron spectroscopy}

The IMFPs are derived from the measurement of the electron elastic backscattering probability ratios from the sample and the reference material and the relevant simulations of electron transport in a solid using the MC algorithm described elsewhere $[2,24]$. The present calculations are performed using the soft- 
ware EPESWIN [25] and differential elastic scattering cross-sections of electrons taken from the database NIST 3.1 [26].

The EPESWIN MC simulations are performed for the set of electron energies and geometry of measurement applying the Ag reference sample with the so-called "recommended" IMFPs for Ag (Ref. [2], Table 10), resulting in the calibration curves. Beside, the MC simulations require a set of input parameters describing the investigated samples, i.e. the surface atomic composition and the density. These parameters have been already evaluated and presented previously (Ref. [10], Table 1). The MC model used in the EPESWIN simulations does not account for recoil effect and surface excitations. These corrections are applied to the measured intensity ratios as described in the sections below.

\section{Recoil effect}

The recoil effect is defined as quasi-elastic scattering of electrons on an atomic core potential. The classical approach for explaining and describing the recoil effect was given by Börsch et al. [11]. The quasi-elastic scattering of electron of given kinetic energy, $E$, on atom characterized by atomic number, $Z$, results in a shift of the energy of the elastic peak position, $\Delta E$ (energy loss) [12], and energy broadening of the elastic peak $[13,14]$. This energy loss, $\Delta E$, is proportional to the incident energy, $E$, the mass of the electron, $m$, and $\sin ^{2}\left(\theta_{0} / 2\right)$ (where $\theta_{0}$ is the electron scattering angle), and inversely proportional to the atomic mass of the scattering atom, $M$ [11]. The energy broadening, i.e. the Doppler broadening due to thermal motion of the scattering atoms is proportional to $\sin \left(\theta_{0} / 2\right)(E m / M)^{1 / 2}$ and temperature, $T^{1 / 2}$, as well as the thermal vibration energy of the lattice atoms $[11,13,14]$. The validity of the Börsch et al. formula was verified experimentally [11-14]. A study of the recoil shift in $\mathrm{Cu}, \mathrm{Ag}$, and Au using 250-3000 eV electrons has been published by Laser and Seah [12].

In the case of high atomic number scattering atom, energy loss, $\Delta E$, and energy broadening are negligible for electron energies ranging between $200 \mathrm{eV}$ and $3000 \mathrm{eV}$ [11]. For the multicomponent compounds, different recoil energy losses and energy broadenings for the constituent atoms, especially those with very different atomic numbers $Z$, modify the shape of the elastic peak [13]. The total cross-section for electron elastic scattering on atom varies approximately as the square of the atomic number, $Z$, and the effect of multiple scattering by different atoms cannot be neglected [14]. These effects can be important for experimental determining of the IMFPs using the EPES method, especially when polymers of high hydrogen content and samples containing elements of varying atomic number range are investigated.

The correction to elastic peak due to the recoil effect on hydrogen is evaluated by Monte Carlo simulation. The code is an extended version of a simple code developed for electron backscattering simulation in general [27]. The original code calculates the scattering angles for each elastic scattering separately. The extension of that also calculates the energy transferred from the electron to the recoiled atom 
because of the momentum exchange. The extension also includes a thermal term for the simulation of thermal energy of the recoiled atoms. It means a given kinetic energy of the atoms before scattering event resulting in a slight change in the transferred energy. Because of these modifications, the energy of those electrons that participate only in elastic scattering differs from their original energy.

The recoil correction is based on the fact that the recoil energy for $\mathrm{H}$ atom is significantly larger than the recoil energy on the other target material atoms, where the atomic number $Z>5$. Thus, this results in the splitting of the elastic peak in the electron spectra into two parts - elastic peak due to scattering on $\mathrm{H}$ atoms and elastic peak due to scattering on atoms of higher atomic numbers.

Instead, these electrons generate a low and wide "heap" in the usual electron spectra. The Monte Carlo simulation can provide the ratio of this $\mathrm{H}$-heap relative to the usual elastic peak. The task of a recoil correction term $k_{\mathrm{r}}(E)$ is to eliminate the elastic peak reduction because of H-heap. Thus the term can be calculated by calculating the full elastic peak $I_{\mathrm{MC}}(E)$, normalized to the one reduced by H-recoil $I_{\mathrm{MC}-\mathrm{r}}(E)$ :

$$
k_{\mathrm{r}}(E)=\frac{I_{\mathrm{MC}}(E)}{I_{\mathrm{MC}-\mathrm{r}}(E)} .
$$

For the present analysis, the corrections $k_{\mathrm{r}}(E)$ were taken from Ref. [27]. Though the definition of recoil correction is simply theoretical, it also needs to have some practical consideration. The $\mathrm{H}$ contribution is not separated from the other element contributions at primary energies below $1 \mathrm{keV}$. Consequently, no correction is needed in this case for recoil.

To evaluate the IMFPs corrected for the recoil effect on hydrogen the measured values of elastic backscattering probabilities, $I_{\exp }(E)$, should be multiplied by the correction factor, $k_{\mathrm{r}}(E)$. Then, the corrected on recoil effect IMFPs are derived from the EPESWIN simulated calibration curves neglecting the recoil effect.

\section{Surface excitations}

Several methods have been proposed for the surface excitation correction [15-23]. The surface excitation is described by the surface excitation parameter $(\mathrm{SEP}), P_{\mathrm{s}}\left(E, \theta_{\mathrm{in}}, \theta_{\text {out }}\right)$, that is defined by the number of surface plasmon excitations experienced by an electron with an energy $E$, impacting the surface at an incidence angle, $\theta_{\text {in }}$, and leaving the surface at an emission angle, $\theta_{\text {out }}$, with respect to the surface normal [15-23]. Then, the SEP consists of the incoming, $P_{\text {in }}$, and the outgoing, $P_{\text {out }}$, contributions

$$
P_{\mathrm{s}}\left(E, \theta_{\text {in }}, \theta_{\text {out }}\right)=P_{\text {in }}\left(E, \theta_{\text {in }}\right)+P_{\text {out }}\left(E, \theta_{\text {out }}\right) .
$$

For free-electron materials the simple expression of Oswald [20] can be applied to determine the SEP parameter. The Oswald [20] equation modified by Werner [21] can be written as

$$
P_{\mathrm{s}}\left(E, \theta_{\text {in }}, \theta_{\text {out }}\right)=\frac{1}{0.173 a_{H} \sqrt{E} \cos \theta_{\text {in }}+1}+\frac{1}{0.173 a_{H} \sqrt{E} \cos \theta_{\text {out }}+1},
$$


where $a_{H}=a / a_{\mathrm{NFE}}$ and $a_{\mathrm{NFE}}=\left(8 a_{0} / \pi^{2} e^{2}\right)^{1 / 2}\left(a_{0}=0.52 \AA\right.$ is a Bohr radius and $e^{2}=14.4 \mathrm{eV} \AA$ is the elementary charge squared) [22]. The parameter $a$ is expressed in units of NFE (the nearly free-electron material), where $a_{\mathrm{NFE}}=$ $0.173 \mathrm{eV}^{-1 / 2}$ and $a_{H}$ is a dimensionless material parameter. For the nearly free-electron materials the value of parameter $a_{H}$ is close to unity, whereas for other materials is different. The value of $a_{H}$ can be estimated for any material from the approximate expression proposed by Werner et al. [22], resulting from the linear fit

$$
a_{H}=0.039 E_{\mathrm{p}}+0.4,
$$

where the constant 0.039 is expressed in $\mathrm{eV}^{-1}$ and $E_{\mathrm{p}}$ (in $\mathrm{eV}$ ) is the generalized plasmon frequency, where

$$
E_{\mathrm{p}}=28.8\left(\frac{N_{\mathrm{v}} \rho}{M}\right)^{1 / 2} .
$$

In Eq. (4b) $N_{\mathrm{v}}$ is the number of valence electrons, $\rho\left(\mathrm{in} \mathrm{g} \mathrm{cm}^{-3}\right.$ ) is the density, $M$ is the atomic mass and the constant 28.8 is expressed in $\mathrm{eV} \mathrm{mol}-1 / 2 \mathrm{~cm}^{3 / 2}$. For evaluating the surface excitation corrections Chen [23] proposed the following formula:

$$
P_{\mathrm{s}}\left(E, \theta_{\text {in }}, \theta_{\text {out }}\right)=\frac{a_{\mathrm{ch}}}{\cos \theta_{\text {in }} \sqrt{E}}+\frac{a_{\mathrm{ch}}}{\cos \theta_{\text {out }} \sqrt{E}},
$$

where $a_{\mathrm{ch}}$ is a fitting parameter called Chen's material parameter. Values of $a_{\mathrm{ch}}$ for selected elements have been proposed [23]. Otherwise, the material parameters by Werner et al. [22], $a_{H}$, and by Chen [23], $a_{\mathrm{ch}}$, for any multicomponent material can be estimated from the EPES [18, 19, 28] and reflection electron energy loss spectroscopy (REELS) methods [19]. For correcting the IMFPs due to surface excitation the measured intensity ratios are multiplied by the reciprocal of the surface excitation correction factor, $f_{\mathrm{s}}$ :

$$
f_{\mathrm{s}}=\frac{\exp \left(-P_{\mathrm{s}-\mathrm{sa}}\right)}{\exp \left(-P_{\mathrm{s}-\mathrm{st}}\right)},
$$

where $P_{\mathrm{s}-\mathrm{sa}}$ and $P_{\mathrm{s}-\mathrm{st}}$ are the surface excitation parameters for the investigated sample and the standard material, respectively. The surface excitation parameters for polythiophenes investigated in the present work are calculated from Eq. (5) using Chen's material parameters, $a_{\mathrm{ch}}$, evaluated by the EPES method [28], i.e.: POT -1.25 , PDOBT -1.25 , PDDoBT -2.5 . For the Ag standard Chen's material parameter, $a_{\mathrm{ch}}=2.34$, was applied [23].

\section{Experimental}

A detailed description of the sample synthesis procedure was given elsewhere [10]. Three polythiophenes: (i) poly(3-octylthiophene), denoted as POT $\left(\mathrm{C}_{24} \mathrm{~S}_{2} \mathrm{H}_{36}\right)$, (ii) poly $\left(4,4^{\prime}\right.$-dioctyl-2,2'-bithiophene), denoted as PDOBT $\left(\mathrm{C}_{24} \mathrm{~S}_{2} \mathrm{H}_{36}\right)$, and (iii) poly (4,4'-didodecyl-2,2'-bithiophene), denoted as PDDoBT $\left(\mathrm{C}_{32} \mathrm{~S}_{2} \mathrm{H}_{52}\right)$, were investigated. The physical properties of poly(alkylthiophenes) strongly depend on their structure [29]. The POT sample was prepared using a 
modification of the Sugimoto et al. method [30] and the PDOBT and PDDoBT samples were prepared following the procedure described elsewhere [31, 32].

The helium pycnometry method for bulk density determination in porous materials, described in detail by Presz et al. [33], was previously applied for determining the bulk density of conductive polymers [6-9] and investigated polythiophenes (Ref. [10], Table 1).

The ESA-31 spectrometer equipped with a high luminosity and high resolution analyser was applied [34]. The electron gun (type VG LEG62, V.G. Scientific, U.K.) operates in the energy range $200-5000 \mathrm{eV}$ with an emission current of $1 \mu \mathrm{A}$, with a defocused beam current on the sample of 1-20 nA, and a beam diameter of about $1.5 \mathrm{~mm}$. An $\mathrm{Ar}^{+}$ion sputtering gun (type AG-21, V.G. Scientific, U.K.) was used to clean the surface of the Ag standard. For the EPES measurements it operated with an $\mathrm{Ar}^{+}$ion current of about 8-20 $\mu \mathrm{A}$ and a beam energy of $2 \mathrm{keV}$.

The angle of X-ray incidence $\left(\mathrm{Al} K_{\alpha}\right)$ of $70^{\circ}$ and the emission angle of the photoelectrons of $0^{\circ}$ relative to the surface normal were used. Survey and narrow scan XPS spectra (C $1 s, \mathrm{~S} 2 p, \mathrm{O} 1 s, \mathrm{Cl} 2 p, \mathrm{Si} 2 p$, and $\mathrm{Na} 1 s)$ were recorded for the polymer samples before and after the EPES analysis to verify that their surface compositions had not changed due to electron beam exposure. After $\mathrm{Ar}^{+}$ sputtering, no carbon or oxygen peaks were detected in the XPS spectra of the Ag standard.

Backscattered electron spectra were recorded in the fixed retardation ratio (FRR) mode to reduce the full width at half maximum (FWHM) of the elastic peak (which includes the energy width of the beam from the electron gun). EPES analysis was carried out after the XPS measurements. The intensities of electrons elastically backscattered from the sputtered $\mathrm{Ag}$ standard and from the polythiophenes were measured sequentially using a primary-electron incidence angle of $50^{\circ}$, and an emission angle of the detected backscattered electrons of $0^{\circ}$, with respect to the surface normal [10]. The energies of the primary electrons were: $200 \mathrm{eV}$, $500 \mathrm{eV}, 1000 \mathrm{eV}, 2000 \mathrm{eV}, 3000 \mathrm{eV}, 4000 \mathrm{eV}$, and $5000 \mathrm{eV}$. The half-cone angles accepted by the analyser (which depend on the retarding ratio) were $5.3^{\circ}, 2.6^{\circ}$, $2.6^{\circ}, 1.7^{\circ}, 2.1^{\circ}, 1.9^{\circ}$, and $1.7^{\circ}$, respectively, and the respective FWHM values varied between $0.4 \mathrm{eV}$ and $0.6 \mathrm{eV}$.

\section{Results \\ 6.1. Surface composition by XPS}

Quantitative XPS analysis of the investigated polythiophenes using the relative sensitivity method [35] and correction parameters, was described in detail elsewhere [10]. Then, the concentration values were corrected for the presence of hydrogen, by assuming the $\mathrm{C}: \mathrm{H}$ ratios to be the same as for the nominal polymer stoichiometry. Beside expected $\mathrm{C}, \mathrm{S}$, and $\mathrm{H}$ content, contaminations of $\mathrm{O}, \mathrm{Cl}, \mathrm{Si}$, and $\mathrm{Na}$ were observed in the XPS spectra. 


\subsection{Determination of IMFPs corrected for hydrogen recoil and surface excitations}

The MC evaluated correction factors due to recoil effect on hydrogen, $k_{\mathrm{r}}(E)$, was observed for all investigated polymers in the electron kinetic energy range $1000-5000 \mathrm{eV}$. This effect was more pronounced for polymers of larger hydrogen content. For POT and PDOBT samples the correction factor, $k_{\mathrm{r}}(E)$, changes in the range 1.018-1.020 and 1.018-1.021, respectively. For the PDDoBT sample the relevant correction factor varies between 1.021 and 1.025, increasing with the electron kinetic energy.

First, the set of IMFPs as a function of electron energy resulting from the measured uncorrected intensity ratios, $I_{\exp }(E)$, and the MC simulated EPESWIN intensity ratios, $I_{\mathrm{MC}}(E)$, were evaluated. Such IMFPs refer to the uncorrected
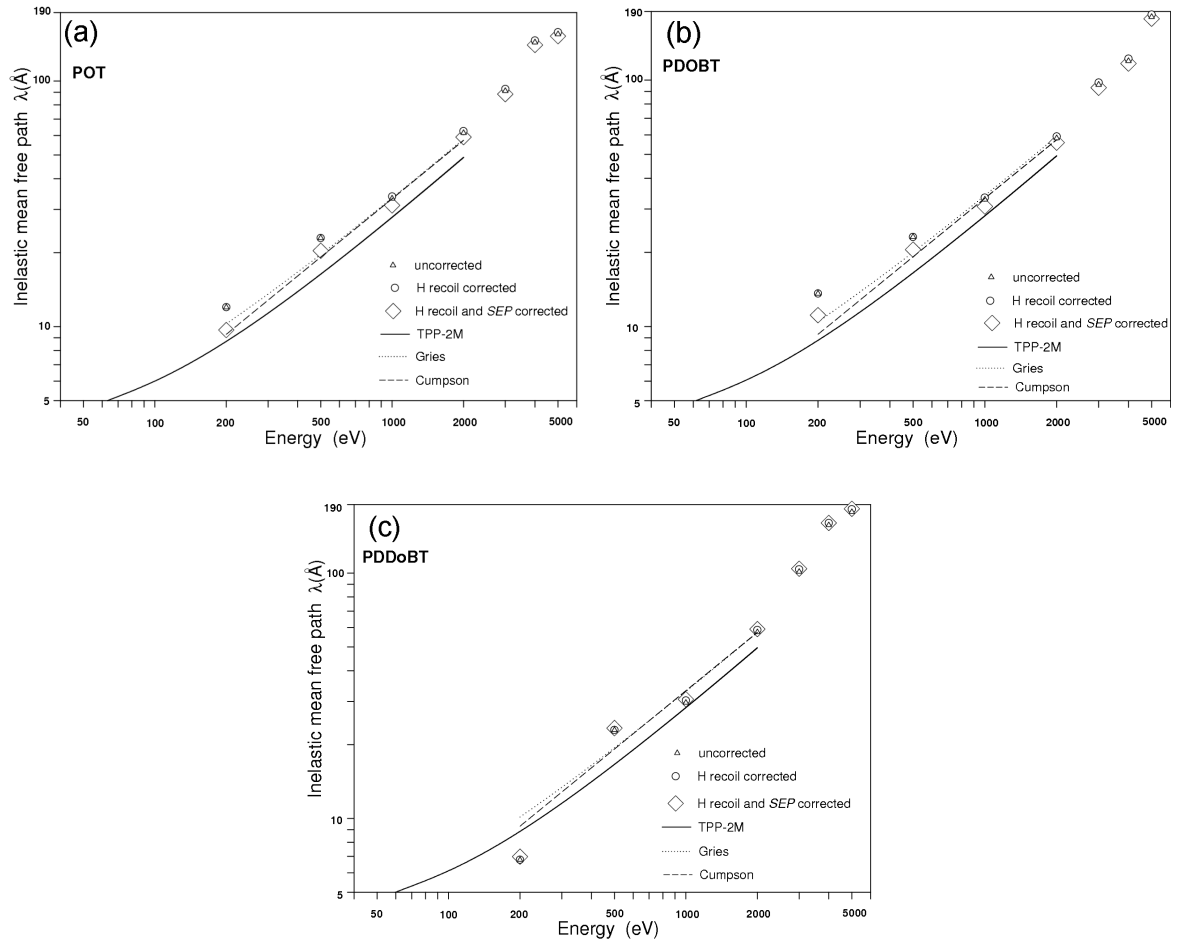

Fig. 1. Comparison of energy dependence of the measured IMFPs in polythiophenes including the IMFPs corrected for hydrogen recoil and surface excitations. Triangle: measured IMFPs. Circle: measured IMFPs corrected for hydrogen recoil. Diamond: measured IMFPs corrected for hydrogen recoil and surface excitations. Solid line: the IMFPs resulting from the TPP-2M predictive formula [3]. Dotted line: the IMFPs resulting from the G1 predictive formula of Gries [4]. Dashed line: the IMFPs resulting from Cumpson [5]. (a) POT, (b) PDOBT, (c) PDDoBT. 
values. Then, the measured intensity ratios were recalculated (multiplication by the correction factor due to recoil effect, $k_{\mathrm{r}}(E)$ ) in order to include the correction due to hydrogen recoil. The set of IMFPs evaluated from corrected on hydrogen recoil intensity ratios results in slightly larger IMFPs for all three polymers. The surface excitation correction of the measured intensity ratios requires multiplication by the reciprocal of the surface excitation correction factor, $f_{\mathrm{s}}$ (Eq. (6)). This correction is applied to the measured intensity ratios after correcting the measured intensity ratios for the hydrogen recoil effect. Such IMFPs are smaller than the uncorrected IMFPs for the POT and the PDOBT sample, and larger in the case of the PDDoBT sample.

Comparison of the IMFPs for three polythiophenes resulting from the EPES method (uncorrected, corrected for hydrogen recoil effect and surface excitations) to the predictive TPP-2M [3], Gries [4], and Cumpson [5] formulae is shown in Fig. 1a-c.

\subsection{Magnitudes of corrections to measured IMFPs}

To determine the magnitudes of the corrections to the measured IMFPs, the parameters of Powell and Jablonski [2] were applied

$$
\operatorname{RMS}=\left[\left(\frac{1}{r}\right) \sum_{j=1}^{r}\left(\lambda_{x}^{j}-\lambda_{\text {ref }}^{j}\right)^{2}\right]^{1 / 2}, \quad R=100\left(\frac{1}{r}\right) \sum_{j=1}^{r}\left|\frac{\lambda_{x}^{j}-\lambda_{\text {ref }}^{j}}{\lambda_{x}^{j}}\right|,
$$

where RMS denotes the root-mean-square deviation, $R$ - the percentage deviation, $\lambda_{x}^{j}$ - the IMFPs found from the EPES measurements at a particular electron energy, $\lambda_{\text {ref }}^{j}$ - a reference IMFPs at the same energy, and $r$ - the number of IMFPs in the calculations. Values of RMS and $R$ for comparison of EPES uncor-

TABLE I

Comparison of scatter due to hydrogen recoil and surface excitation corrections, expressed as the RMS and the mean percentage $(R)$ deviations (Eq. (7)). Energy range 200-5000 eV. Number of the IMFPs $r=7$.

\begin{tabular}{l|cl|c|c}
\hline \hline Sample & \multicolumn{2}{|c|}{ Compared EPES IMFPs } & RMS $[\AA]$ & $R[\%]$ \\
\hline POT & uncorrected & H recoil & 1.64 & 1.26 \\
& uncorrected & SEP & 4.05 & 8.01 \\
& uncorrected & H recoil and SEP & 2.66 & 6.82 \\
\hline PDOBT & uncorrected & H corrected & 1.89 & 1.36 \\
& uncorrected & SEP & 4.22 & 7.96 \\
& uncorrected & H recoil and SEP & 2.63 & 6.68 \\
\hline \multirow{2}{*}{ PDDoBT } & uncorrected & H corrected & 2.18 & 1.49 \\
& uncorrected & SEP & 0.63 & 1.21 \\
& uncorrected & H recoil and SEP & 2.64 & 2.65
\end{tabular}


TABLE II

Comparison of scatter between the uncorrected and the corrected for $\mathrm{H}$ recoil and surface excitations EPES IMFPs with the IMFPs resulting from predictive formulae, expressed as the RMS and the mean percentage $(R)$ deviations (Eq. (7)). Energy range 200-2000 eV. Number of the IMFPs $r=4$.

\begin{tabular}{l|c|c|c|c|l}
\hline \hline \multirow{2}{*}{ Sample } & \multicolumn{2}{|c|}{ Uncorrected IMFPs } & \multicolumn{2}{l|}{ Corrected IMFPs } & \multirow{2}{*}{ Reference IMFPs } \\
\cline { 2 - 5 } & RMS $[\AA]$ & $R[\%]$ & RMS $[\AA]$ & $R[\%]$ & \\
\hline POT & 7.77 & 23.20 & 5.78 & 14.45 & TPP-2M [3] \\
& 2.51 & 8.65 & 1.31 & 4.61 & Gries [4] \\
& 3.08 & 11.14 & 1.47 & 4.79 & Cumpson [5] \\
\hline PDOBT & 6.45 & 23.33 & 4.20 & 15.14 & TPP-2M [3] \\
& 2.34 & 10.48 & 2.37 & 6.28 & Gries [4] \\
& 2.95 & 12.80 & 1.83 & 8.41 & Cumpson [5] \\
\hline PDDoBT & 5.21 & 19.10 & 6.01 & 19.82 & TPP-2M [3] \\
& 2.96 & 19.20 & 2.93 & 18.19 & Gries [4] \\
& 2.87 & 16.44 & 2.85 & 15.58 & Cumpson [5]
\end{tabular}

rected IMFPs and EPES IMFPs corrected for hydrogen recoil effect and surface excitations are given in Table I. Similarly, values of RMS and $R$ for comparison of EPES IMFPs uncorrected and corrected for hydrogen recoil effect and surface excitations with the IMFPs resulting from the TPP-2M [3], Gries [4], and Cumpson [5] formulae are given in Table II.

\section{Discussion}

Surface morphology, line scans and values of average surface roughness (between $5.59-19.93 \mathrm{~nm}$ ) reported elsewhere $[18,28]$ show negligible differences among the three polythiophenes. The PDOBT sample, however, seems to be different from the others having the largest surface roughness. As it was shown previously, variations of surface roughness in $\mathrm{Ni}$ [36], Ge [37], and $\mathrm{Si}$ [38] in the range of several hundred $\mathrm{nm}$ do not appreciably affect the elastic backscattering coefficient. This refers to measurements at emission angles of $35^{\circ}$ and $70^{\circ}$ [38]. The helium pycnometry densities for polythiophenes reported elsewhere [10] are similar (around $\left.1 \mathrm{~g} \mathrm{~cm}^{-3}[10]\right)$. For undoped and metal doped polymers the density values vary between $0.9 \mathrm{~g} \mathrm{~cm}^{-3}$ and $1.6 \mathrm{~g} \mathrm{~cm}^{-3}$ [6-10]. Comparison of polythiophenes nominal and XPS composition indicates contamination of $\mathrm{Cl}, \mathrm{Si}, \mathrm{Na}$, and $\mathrm{O}$, resulting from the synthesis environment. The atomic concentrations of $\mathrm{C}$ and $\mathrm{S}$ found from the XPS measurement are similar to the nominal compositions.

Slight increase is observed in EPES measured IMFPs corrected for hydrogen recoil effect in comparison to the uncorrected IMFPs (Fig. 1a-c). The surface excitation corrections result in decreasing the IMFPs for the POT and PDOBT 
samples (Fig. 1a, b). In the primary electron energy range 200-2000 eV, all of the measured IMFPs are closer to Gries [4] and Cumpson [5] IMFPs.

The percentage deviations in the IMFPs due to corrections for hydrogen recoil vary between $1.26 \%$ and $1.49 \%$, whereas the deviations due to surface excitations vary between $1.21 \%$ and $8.01 \%$ (Table I). The deviations due to accounting for both, recoil effect on hydrogen and surface excitations change in the range 2.65$6.82 \%$. These deviations are almost equally important as deviations in IMFPs due to change of composition reported previously from nominal to that obtained from XPS which are $4.51 \%, 3.80 \%$, and $5.23 \%$ for POT, PDOBT and PDDoBT, respectively (Ref. [10], Table 5). This indicates that both corrections to the EPES IMFPs due to hydrogen recoil and surface excitation corrections are not negligible, as well as deviations in the IMFPs due to changes in surface atomic composition. Comparison of the scatter between the measured IMFPs uncorrected and corrected for recoil effect on hydrogen and surface excitations with the predicted IMFPs shows decreasing deviations due to correcting procedures (Table II). The best agreement is achieved between the IMFPs resulting from Cumpson [5] formula (Table II). The largest deviations are observed between the measured IMFPs and the TPP-2M [3] formula IMFPs (Table II). However, the accuracy of determining the uncorrected and corrected electron transport parameters depends on several factors such as the accuracy of the input parameters (composition, density), validity of the model of electron transport, reliability of values of electron elastic cross-sections and accuracy of the material parameters applied in correction procedure. As it has been shown recently by Werner et al. [39], the values of material parameters, $a_{H}$, represent a scatter depending on the method applied for their determination.

The quantitative evaluation of deviations between the IMFPs indicates that the average surface roughness of the polymers reported in the present work is not mostly important. The correction due to hydrogen recoil effect is smaller than the correction due to surface excitations. However, hydrogen and surface excitation corrections to the IMFPs seem to be worth considering when accounting for high accuracy IMFPs evaluated by the EPES method. The results of the present work indicate the importance of correcting the EPES IMFPs for hydrogen recoil effect and surface excitation, especially when considering the polymers of high hydrogen content.

As previously indicated, the IMFP is one of the correction parameters in calculating the surface composition from the AES and XPS intensities. However, in the relative sensitivity factor approach with correction parameters applied in this work for determining the polymer surface composition from the XPS intensities, only the energy dependence of the IMFP is important, rather than the absolute value. It is expected that the variation of the IMFP indicated in the present work due to hydrogen recoil effect and surface excitation has insignificant influence on the calculated surface composition of polymers. However, there are other quantitative applications of the XPS in which the role and accuracy of the IMFPs is 
more pronounced, i.e. overlayer thickness determination, estimation of the analysed thickness. In these cases a given percentage variation of the IMFP has more significant influence on the accuracy of the evaluated results [40, 41].

\section{Acknowledgments}

The authors would like to thank to Dr. L. Kövér (Institute of Nuclear Research of the Hungarian Academy of Sciences) for helpful discussions during preparation of the manuscript, Dr. M. Zagórska and Dr. I. Kulszewicz-Bajer (Faculty of Chemistry, Technical Warsaw University) for synthesis of polythiophenes and Dr. A. Presz (Institute for High Pressure, Polish Academy of Sciences) for the helium pycnometry density measurements of samples. The authors (J.T. and D.V.) thank for the support of Hungarian Research Foundation OTKA T0 38016. The author (B.L.) acknowledges the support of the co-operation contract between the Polish and Hungarian Academy of Sciences (PL-15) and the EU SURPHARE GMA1-2002-72057.

\section{References}

[1] ASTM Standard E673-95c, Annual Book of ASTM Standards, Vol. 3.06, American Society for Testing and Materials, West Conshohocken, PA 1997, p. 907.

[2] C.J. Powell, A. Jablonski, J. Phys. Chem. Ref. Data 28, 19 (1999).

[3] S. Tanuma, C.J. Powell, D.R. Penn, Surf. Interface Anal. 21, 165 (1994).

[4] W.H. Gries, Surf. Interface Anal. 24, 38 (1996).

[5] P.J. Cumpson, Surf. Interface Anal. 31, 23 (2001).

[6] B. Lesiak, A. Kosinski, M. Krawczyk, L. Zommer, A. Jablonski, J. Zemek, P. Jiricek, L. Kövér, J. Tóth, D. Varga, I. Cserny, Appl. Surf. Sci. 144, 168 (1999).

[7] B. Lesiak, A. Kosiński, M. Krawczyk, L. Zommer, A. Jablonski, L. Kövér, J. Tóth, D. Varga, I. Cserny, J. Zemek, P. Jiricek, Polish J. Chem. 74, 847 (2000).

[8] B. Lesiak, A. Jablonski, J. Zemek, M. Trchova, J. Stejskal, Langmuir 16, 1415 (2000).

[9] B. Lesiak, A. Kosinski, A. Jablonski, L. Kövér, J. Tóth, D. Varga, I. Cserny, Surf. Interface Anal. 29, 614 (2000).

[10] B. Lesiak, A. Kosinski, A. Jablonski, L. Kövér, J. Tóth, D. Varga, I. Cserny, M. Zagorska, I. Kulszewicz-Bajer, G. Gergely, Appl. Surf. Sci. 174, 70 (2001).

[11] H. Börsch, P. Wolter, H. Schönebeck, Z. Phys. 199, 124 (1967).

[12] D. Laser, M.P. Seah, Phys. Rev. B 47, 9836 (1993).

[13] A. Sulyok, G. Gergely, M. Menyhard, J. Tóth, D. Varga, L. Kövér, Z. Berenyi, B. Lesiak, A. Kosinski, Vacuum 63, 371 (2001).

[14] D. Varga, K. Tökési, Z. Berényi, J. Tóth, L. Kövér, G. Gergely, A. Sulyok, Surf. Interface Anal. 31, 1019 (2001).

[15] S. Tanuma, S. Ichimura, K. Goto, J. Surf. Anal. 5, 48 (1999).

[16] S. Gurban, G. Gergely, M. Menyhard, J. Adam, M. Adamik, Cs. Daroczi, J. Tóth, D. Varga, A. Csik, B. Gruzza, Surf. Interface Anal. 34, 206 (2002). 
[17] J. Zemek, P. Jiricek, B. Lesiak, A. Jablonski, Surf. Sci. 531, L335 (2003).

[18] G. Gergely, M. Menyhard, B. Lesiak, A. Kosiński, R. Nowakowski, A. Jablonski, J. Tóth, D. Varga, Appl. Surf. Sci., in press.

[19] G. Gergely, Prog. Surf. Sci. 71, 31 (2002).

[20] R. Oswald, Ph.D. thesis, University of Tübingen, 1992.

[21] W.S.M. Werner, Surf. Interface Anal. 31, 141 (2001).

[22] W.S.M. Werner, W. Smekal, Ch. Tomastik, H. Störi, Surf. Sci. Lett. 461, 486 (2001)

[23] Y.F. Chen, Surf. Sci. 519, 115 (2002).

[24] A. Jablonski, P. Jiricek, Surf. Sci. 412/413, 42 (1998).

[25] A. Jablonski, Surf. Interface Anal. 37, 1035 (2005).

[26] A. Jablonski, F. Salvat, C.J. Powell, NIST Electron Elastic-Scattering Cross-Section Database, Version 3.1, Standard Reference Data Program, Database 64, National Institute of Standard and Technology, Gaithersburg, MD 2003; http://www.nist.gov/srd/nist64.html.

[27] G.T. Orosz, A. Sulyok, G. Gergely, S. Gurban, M. Menyhard, Microsc. Microanal. 9, 343 (2003).

[28] G. Gergely, J. Tóth, M. Menyhard, D. Varga, S. Gurban, A. Sulyok, A. Kosinski, B. Lesiak, A. Jablonski, R. Nowakowski, J. Electron Spectrosc. Relat. Phenom., submitted for publication.

[29] J. Roncali, Chem. Rev. 92, 711 (1992).

[30] R. Sugimoto, S. Takeda, M.V. Gu, K. Yoshino, Chem. Express 1, 635 (1986).

[31] M. Zagórska, M. Krische, Polymer 31, 1379 (1990).

[32] M. Zagórska, I. Kulszewicz-Bajer, A. Pron, P. Barta, F. Cacialli, R.H. Friend, Synth. Met. 101, 142 (1999).

[33] A. Presz, M. Skibska, M. Pilecki, Powder Handling and Processing 7, 321 (1995).

[34] L. Kövér, D. Varga, I. Cserny, J. Tóth, K. Tökési, Surf. Interface Anal. 19, 9 (1992).

[35] J.F. Moulder, W.F. Stickle, P.E. Sobol, K.D. Bomben, Handbook of X-ray Photoelectron Spectroscopy, Perkin Elmer Co., Eden Prairie, Minnesota 1992.

[36] P. Jiricek, J. Zemek, P. Lejćek, B. Lesiak, A. Jablonski, M. Ċerñanský, J. Vac. Sci. Technol. A 20, 447 (2002).

[37] B. Lesiak, A. Jablonski, J. Zemek, P. Jiricek, J. Pavluch, Surf. Interface Anal. 33, 381 (2002).

[38] J. Zemek, P. Jiricek, A. Jablonski, B. Lesiak, Surf. Interface Anal. 34, 215 (2002).

[39] W.S.M. Werner, L. Kövér, S. Egri, J. Tóth, D. Varga, Surf. Sci. 585, 85 (2005).

[40] A. Jablonski, C.J. Powell, Surf. Sci. Rep. 47, 33 (1992).

[41] C.J. Powell, A. Jablonski, W.S.M. Werner, W. Smekal, Appl. Surf. Sci. 239, 470 (2005). 